\title{
Rainer Wimmer (Mannheim)
}

\section{Chancen der Sprachkritik}

\section{Vorbemerkungen zu Sinn und Aufgaben der Sprachkritik}

Sprachkritik soll und kann die Freiheit stärken. Die Chancen für eine vernünftige Sprachkritik verbessern heißt zugleich, die Chancen der Freiheit verbessern. Wer sich in den Dienst einer so allgemeinen These stellt, die zudem ein so großes und altehrwürdiges Thema wie das der Freiheit aufgreift, muß natürlich auf eine Fülle von kritischen Fragen gefaßt sein. Einige solcher Fragen möchte ich vorab formulieren. Ich werde sie natürlich nicht alle beantworten können, und wo ich zu Antworten kommen kann, werden diese noch sehr unzulänglich, in jedem Fall unvollständig sein.

Eine erste und wohl auch die grundsätzliche Frage ist: Wie geht das Thema Sprache in der Weise mit dem Thema Freiheit zusammen, wie ich es annehme? Inwiefern kann Arbeit an der Sprache - und ich sehe Sprachkritik als konstruktive Arbeit an - die Freiheit des einzelnen Menschen stärken? - Viele Erfahrungen zeigen, daß man mit Sprache alles mögliche machen kann, was Menschen hilft oder was ihnen schadet. Insbesondere kann man die Sprache auch benutzen, um andere zu manipulieren, zu beherrschen und zu unterdrücken. In Diktaturen werden die sprachlichen Mittel ausgenützt, um Macht zu festigen, Freiheiten einzuschränken, Lebensmöglichkeiten abzuschneiden. Wie also kann man annehmen, daß sprachkritische Arbeit und - wie ich darüber hinaus noch sagen möchte - jegliche Arbeit an der Sprache, die diesen Namen verdient, ein Element der Freiheit bei sich hat und der Erweiterung menschlicher Handlungsmöglichkeiten dient, auch wenn das nicht immer auf Anhieb augenfällig ist und dieser Nutzen sich zuweilen auch erst längerfristig einstellt? Auf diese Frage werde ich später eine Antwort versuchen, indem ich einige sprachtheoretische Überlegungen anstelle. Ich glaube nämlich, daß man aus der Sprachtheorie, der Theorie über die allgemeine Sprachfähigkeit, also der Theorie über die langage, nicht über die langue (eine Einzelsprache) Aussagen über ein allen natürlichen Sprachen inhärentes analytisches Potential ableiten kann, das in bezug auf die Freiheitsfrage bzw. in bezug auf die Gefahr einer Herrschaft durch Sprache eine gewissermaßen selbstheilende Kraft mit sich bringt.

Eine zweite Frage, die durch meine allgemeine These, Sprachkritik diene der Freiheit des einzelnen, aufgeworfen wird, ist: Was ist hier unter Sprachkritik zu verstehen? Welche Art von Sprachkritik könnte denn überhaupt eine solche Leistung erbringen, die hier verlangt wird? - Es gibt eine 
Art philosophischer Sprachkritik, die aufs Ganze geht, insofern sie - unter der Voraussetzung, daß alle Erkenntnis sprachlich produziert und vermittelt wird - durch die Sprache hindurch Erkenntniskritik will. Diese Art radikaler Sprachkritik ist alt. Sie bestimmt bereits die bekannte Idolenlehre des Francis Bacon zu Beginn der Neuzeit. In neuerer Zeit kann sie sich insbesondere auf Fritz Mauthners Kritik der Sprache berufen. ${ }^{1}$ Mauthner wandte sich gegen jegliche Verfestigung von Erkenntnissen in sprachlichen $\mathrm{Mu}$ stern und Formen. Er wollte mit seiner Sprachkritik alles aus dem Wege räumen, was dem ständigen Wandel von Erkenntnissen entgegenstehen könnte, und er erhob daher die Auflösung aller verfestigten und standardisierten Benennungen- und Prädikationsstrukturen zum Prinzip. Mauthner schreibt (im 1. Bd. seiner „Beiträge zu einer Kritik der Sprache“, S. 713):

So ist es die Sprache allein, die

für uns dichtet und denkt, die uns auf einiger Höhe die Fata Morgana

der Wahrheit oder der Welterkenntnis vorspiegelt, die uns auf der steilsten Höhe losläßt und uns zuruft: Ich war dir ein Führer! Befreie dich von mir! Die Kritik der Sprache muß Befreiung von der Sprache als höchstes Ziel der Selbstbefreiung lehren. Die Sprache wird zur Selbstkritik der Philosophie. Diese selbstkritische Philosophie wird durch ihre Resignation nicht geringer als die alten selbstgerechten Philosophien. Denn von der Sprache gilt wie von jedem anderen Märtyrer der Philosophie das tapfere Wort: Qui potest mori, non potest cogi.

Die Sprachkritik, die ich mir vorstelle, hat nicht die philosophischen und erkenntnistheoretischen Ambitionen und nicht die radikale Tendenz einer Fundamentalkritik, wie wir sie bei Mauthner finden. Ich denke auch nicht an eine Sprachkritik auf der Basis einer Logik, die natürlichsprachliche Phänomene an den Konstrukten einer wie immer gearteten Kunstsprache mißt und bewertet.

Vielmehr schwebt mir eine Art von Sprachkritik vor, die als Teil der Sprachwissenschaft im herkömmlichen Sinne fungieren kann, insofern sie

1. einzelsprachliche Phänomene in einer spezifischen Auswahl zu ihrem Gegenstand hat und diese mit den Mitteln der linguistischen Syntax und Semantik (einschließlich Pragmatik) untersucht,

2. alle sprachtheoretischen Aussagen, die über die Beschreibung einer Einzelsprache hinausreichen, auf empirische Befunde einer Einzelsprache stützt und

1 Vgl. Mauthner ${ }^{3} 1923$. 
3. alle Bewertungen von sprachlichen Phänomenen und insbesondere von sprachlichen Handlungsmustern aus sprachwissenschaftlichen Analysen ableitet.

Die Sprachkritik ist also in erster Linie Sprachanalyse, und sie ist Kritik an Sprechern und deren Sprachverwendung nur insofern, als sie Sprechern zu denken geben kann und sie vielleicht auch zu Änderungen ihres Sprachgebrauchs anregen kann. Ich verwende den Terminus Kritik hier also in der traditionellen Verwendungsweise der Aufklärung, wo beispielsweise bei Kant „Kritik der reinen Vernunft“ die Analyse der reinen Vernunft meint. ${ }^{2}$

Für die Sprachkritik, die ich mir vorstelle, möchte ich zunächst einige kleinere Beispiele geben, um dann Konsequenzen zu ziehen. Die Beispiele sind zugegebenermaßen recht einfach, und sie sollen es auch sein. Sie beziehen sich ferner nur auf einen einzigen Komplex sprachlicher Phänomene, den man unter der Überschrift zusammenfassen könnte: Was kann man bei der Verwendung von komplexen nominalen Ausdrücken alles mitmeinen und stillschweigend voraussetzen, ohne es explizit zu machen?

\section{Beispiele für komprimierten Sprachgebrauch}

Das Phänomen, um das es in meinen Beispielen geht, ist auch von Bundeskanzler Helmut Kohl in seiner Rede zur Eröffnung der Frankfurter Buchmesse 1984 angesprochen worden. Ich darf aus der Rede des Bundeskanzlers zitieren. ${ }^{3}$

Auch die Sprache der Politik kennt Fluchtbewegungen: Es gibt die Flucht in hektischen Wortreichtum, um nichts mitzuteilen. Es gibt die Flucht in politische Sprachspiele. Da werden Begriffe besetzt, umgedeutet, konstruiert, aufgebläht, demontiert. Der Kampf um Worte gerät zum Machtkampf.

„Friedenskampf“, „gewaltfreier Widerstand“, „Ziviler Ungehorsam“ sind Beispiele absichtsvoll gewählter Mehrdeutigkeit. Aussage und Dementi sind bewußt miteinander verwoben.

Die Ausuferung politischer Schlüsselbegriffe macht sie beliebig handhabbar, macht es möglich, mit ihnen sowohl prinzipiellen Widerspruch wie auch die Illusion von Übereinstimmung in Worte zu fassen.

Wenn ich recht verstehe, soll mit einem Beispiel wie Friedenskampf demonstriert werden, daß hier in einem einzigen nominalen Ausdruck in gewissermaßen kondensierter Form ein Widerspruch enthalten sei, nämlich: Die beiden Aussagen „Es ist Friede“ und „Es herrscht Kampf“ gingen nicht zusammen, stellten einen Widerspruch dar. Der Kanzler hat diese Erscheinung als ,absichtsvoll gewählte Mehrdeutigkeit“ charakterisiert. Von Mehrdeutigkeit im landläufigen Sinne kann sicher nicht die Rede sein. Aber es

2 Vgl. auch Wimmer 1984, 16.

3 Zitiert nach der Pressemitteilung der Bundesregierung Nr. 503/84 vom 2. Oktober 1984. 
erscheint plausibel, für das Beispiel eine Analyse vorzuschlagen, die die beiden Komponenten des Wortes Friedenskampf trennt und expliziert. Freilich sind auf diesem Wege ganz verschiedene Analyseergebnisse denkbar: Nicht für jedermann müssen Kampf und Friede absolute Gegensätze darstellen. Das hängt von den Bedeutungen der Komponentenwörter ab, d. h. von den Kommunikationsgeschichten, die die Sprecher mit diesen Wörtern verbinden. Und das hängt davon ab, wie die Relation zwischen den Komponentenwörtern verstanden wir. Ein christliches Mitglied der Friedensbewegung wird unter „Kampf für den Frieden“ eher harte Arbeit für den Frieden verstehen - ohne jegliche Gewaltanwendung - als kämpferisch-gewaltsames Eintreten für bestimmte Ideen und Ziele. Es gibt nicht das eine und allein richtige Verständnis eines Kompositums wie Friedenskampf.

\section{BEISPIEL 1}

Atom, atomare Abschreckung, Atombombe, Atombrennstoff, atomare Diplomatie, Atom-Drohung, Atomenergie, atomare Entsorgung, atomare Garantie, atomare Geiseln, Atomgeiseln, atomare Habenichtse, Atomhabenichtse, Atomklub, atomares Komplicentum, Atomkraftwerk, atomare Maginot-Linie, atomare Mauer, Atommeiler, atomare Parität, atomares Patt, Atompatt, Atom-Pool, Atomschirm, atomare Schwelle, Atomschwelle, atomare Sicherheit, Atomspirale, atomarer Störfall, atomare Strahlenbelastung, atomare Verseuchung, Atomwaffe, atomwaffenfreie Zone, Atomwaffensperrvertrag, atomarer Zwerg.

Die als Beispiel 1 gegebene Liste enthält nominale Ausdrücke mit dem Lexem Atom, die in den vergangenen dreißig Jahren als politische Schlagwörter und Kampfbegriffe mehr oder weniger „Karriere“ gemacht haben. ${ }^{4}$ Diese Ausdrücke können Gegenstand sprachkritischer Analysen sein, insofern hinter ihnen semantische Kämpfe als Kommunikationsgeschichten stehen, die es wenigstens teilweise zu rekonstruieren gilt, wenn man sie verstehen bzw. mit Erfolg gebrauchen will. Für nicht mit den jeweiligen Kommunikationsgeschichten vertraute Sprecher haben die inhaltlichen Komprimierungen in den Ausdrücken etwas Hermetisches, das einer sprachkritischen Brechung bedarf, wenn diese Teile des Wortschatzes nicht nur dem Gebrauch in bestimmten gesellschaftlichen Gruppen vorbehalten bleiben sollen.

Nehmen wir das Beispiel Atombrennstoff: Welche Implikationen, die in dem Wort verpackt sind, müßte eine linguistische Analyse versuchen, zutage zu fördern? Unter anderen sicherlich die euphemistische Aussage, daß es sich bei dieser Sache um einen Brennstoff handele wie etwa Holz, Kohle, Öl und ähnliche Dinge. Weiterhin sicherlich die Implikation, daß man diese Sache entsprechend genauso gut handhaben, verarbeiten könne wie die

4 Vgl. Nunn 1974. 
einfachen „wirklichen“ Brennstoffe. Weiterhin die Implikation, daß die Rückstände eines solchen Brennstoffs sich vergleichen ließen mit den Verbrennungsrückständen von Holz, Kohle oder Öl. - Solche Analysen wären kritische Wort- und Kommunikationsgeschichten, und einige solcher Analysen hat die semantisch-pragmatische Linguistik auch bereits vorgelegt, insbesondere in der letzten Zeit im Zusammenhang mit dem Jahr 1984 des großen Bruders. ${ }^{5}$ Die Analysen müßten eigentlich in den Zusammenhang eines größeren zeitkulturellen Wörterbuchs gestellt werden. Doch das wäre natürlich eine immense Aufgabe.

\section{BEISPIEL 2}

Klarheit ist alles, ist Sieg! Klarheit in der Erkenntnis des gewaltigen Streitens kann nur aus der Erkenntnis scharfumrissener Weltanschauung erstehen. Weltanschauung ist halt- und wertlos, wenn sie nicht, dem Uferlosen entrissen, umgrenzter Glaube geworden ist. Nationalsozialistischer Glaube aus völkischer Weltanschauung ist eindeutig, bedeutet Abbruch aller Brücken zu sterbenden, verfaulenden Welten, strebt unbeirrbar der Errichtung einer neuen Welt entgegen, getragen von dem neuen Menschen völkischer Art. Schaffung dieses Menschen ist die erste Tat werdender Erlösung ...

(Völkischer Beobachter 28.10.1926)

Ich zitiere dieses Beispiel nach Peter von Polenz 1981. Das Textstück zeichnet sich dadurch aus, daß im Zentrum eine komprimierte Nominalphrase steht, nämlich die Phrase „nationalsozialistischer Glaube aus völkischer Weltanschauung“, deren komprimierter Inhalt extrem explizierungsbzw. interpretationsbedürftig ist. Der umgebende Text aber kommt der Interpretationsbedürftigkeit überhaupt nicht entgegen; ganz im Gegenteil: Die expliziten Prädikationen bzw. Aussagen sind nichtssagend, schlicht, gemeinplätzig und tragen nichts zur Analyse der zentralen Phrase bei. Alle Prädikationen, die überhaupt gemacht werden, sind wiederum in nominale Ausdrücke implizierend verpackt: Was heißt schon „Klarheit in der Erkenntnis des gewaltigen Streitens“ oder „Abbruch aller Brücken zu sterbenden, verfaulenden Welten“ oder „neuer Mensch völkischer Art“ oder „erste Tat werdender Erlösung"? - Das Ganze ist ein Musterbeispiel für leerformelhaften Textaufbau: Im prädikativ-verbalen Bereich ist der Text äußerst dürftig und kommt meist mit der Kopula ist aus. Das Leseverständnis hangelt sich von Nominalphrase zu Nominalphrase, die jeweils leerformelhaft inexplizit ist.

5 Vgl. Blanc 1983; Schwenger 1983. 


\section{BEISPIEL 3}

„aber das sind wir, da kommen wir her: die brut aus den vernichtungs- und zerstörungsprozessen der metropolengesellschaft, aus dem krieg aller gegen alle, der konkurrenz jeder gegen jeden, des systems, in dem das gesetz der angst, des leistungsdrucks herrscht, des einer-auf-die-kosten-des-andern, der spaltung des volks in männer und frauen, junge und alte, gesunde und kranke, ausländer und deutsche und der prestigekämpfe, und da kommen wir her: aus der isolation im reihenhaus, in den betonsilos der vorstädte, den zellengefängnissen, asylen und trakts. aus der gehirnwäsche durch die medien, den konsum, die prügelstrafen, die ideologie der gewaltlosigkeit; aus der depression, der krankheit, der deklassierung, aus der beleidigung und erniedrigung des menschen, aller ausgebeuteten menschen im imperialismus“.

(Rede von Ulrike Meinhof, 13.9.1974)

Nur noch einige wenige Bemerkungen zum Beispiel 3: Der Text besteht fast ausschließlich aus einer Aneinanderreihung komprimierter Nominalphrasen wie „brut aus den vernichtungs- und zerstörungsprozessen der metropolengesellschaft" oder "ausgebeutete menschen im imperialismus". Der Text ist wegen seiner Inexplizitheit im Grunde nur für einen engen Kreis von Eingeweihten, von In-group-Mitgliedern, zustimmungs- oder ablehnungsfähig, von Eingeweihten, für die die chiffrenhaften Kondensierungen von Erfahrungen in schlagwortartigen Nominalausdrücken eigentlich auch gar keiner Auflösung, keiner Explizierung mehr bedürfen, eben weil gar keine in irgendeiner Weise offene Kommunikation mit den Hörern mehr angestrebt wird. Es geht nur noch um eine Selbstbestätigung ideologisch verfestigter Erfahrungs- und Wissensbestände.

Der Auszug aus der Rede von Ulrike Meinhof ist natürlich ein extremes Beispiel. Mit dem Phänomen, für das es steht, haben wir es aber vielerorts im Sprachgebrauch des Alltags, der Öffentlichkeit, der Politik und auch der Wissenschaft zu tun. Das Phänomen wird den Kommunikationsbeteiligten oft auch mehr oder weniger bewußt, eben wenn kommunikationsgeschichtliche Verfestigungen von Bedeutungen als solche erkannt werden und umstritten sind. In den semantischen Kämpfen, die dann ausbrechen können, geht es jeweils in einem um die Sache und um deren sprachliche Fassung.

\section{Einige sprachtheoretische Überlegungen}

Die Beispiele, die ich angeführt habe, ermöglichen relativ zwanglos einige sprachtheoretische Überlegungen, die ich zu Beginn angekündigt habe. Ich meine sprachtheoretische Überlegungen zur elementaren Struktur von Aussagen in natürlichen Sprachen und zur Komplizierung und Komprimierung von Aussagen. Alle Beispiele haben gemeinsam, daß es galt, in einzelnen nominalen Ausdrücken mitverpackte Aussagen zu explizieren und damit einer Analyse zugänglich zu machen. Die Beispiele exemplifizieren 
damit eine universale Eigenschaft natürlicher Sprachen, mehrere Aussagen zusammenfassend ineinander einbetten zu können, wobei die eingebettete Aussage jeweils etwas aussagt über den einbettenden Ausdruck oder Teile von ihm oder über Gegenstände, die von ihm oder seinen Teilen bezeichnet werden. Die eingebetteten Aussagen erscheinen oft in der Form von Relativsätzen, von Attributen und von Kompositionselementen in nominalen Ausdrücken. Lassen Sie mich den Sachverhalt an einem vielleicht etwas extremen Beispiel erläutern.

\section{BEISPIEL 4}

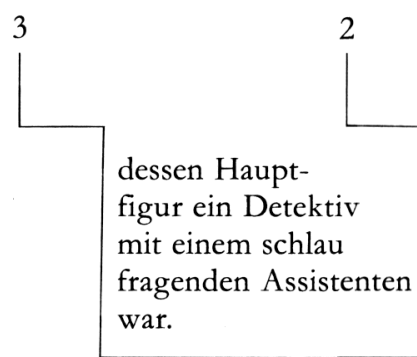

5

Conan Doyle
4

Der Romancier des

Detektivs mit dem schlau fragenden

Assistenten.

Sie finden ganz rechts in dem Beispielschema 4 den nominalen Ausdruck mit drei eingebetteten Aussagen jeweils in der Form eines Relativsatzes. In vier Schritten erscheint die komplexe Phrase dann syntaktisch und semantisch komprimiert und reduziert, bis schließlich nur noch der Eigenname für den ganzen Ausdruck steht. Die syntaktischen und semantischen Reduktionen, die vorgenommen werden, sind vielfältig und sehr komplex. Sie sind sprachwissenschaftlich im einzelnen noch keineswegs hinreichend analysiert, und man kann natürlich auch nicht behaupten, daß der Eigenname 
Conan Doyle dasselbe bedeutet wie der nominale Ausdruck mit den drei eingebetteten Relativsätzen, obwohl in vielen Texten der komprimierteste Ausdruck salva veritate gegen den explizitesten ausgetauscht werden kann. In dem Reduktionsprozeß ist viel zu viel explizites Wissen verloren gegangen, als daß ein Eigenname als gleichbedeutend mit einer komplexen Nominalphrase angesehen werden könnte.

Mir scheint klar, daß natürliche Sprachen beides brauchen: auf der einen Seite Eigennamen und ähnlich komprimierte Ausdrücke, die kommunikationshistorisches Wissen inexplizit und zusammenfassend verdichtet enthalten, und auf der anderen Seite explizite Ausdrücke, in denen die gemeinten Aussagen bzw. Prädikationen mehr oder weniger offen zutage liegen. Eigennamen und eigennamenähnliche Formeln haben die Funktion, überkommenes und verfestigtes und normiertes $W$ issen in komprimierter Form leicht verfügbar zu machen. Explizite Prädikationen dagegen dienen der Neuzuschreibung von Eigenschaften, der kreativen Veränderung von Wissensbeständen, der innovativen Arbeit an Sprache und Welt. ${ }^{6}$

Der Sprachwissenschaftler, Schriftsteller und Erfinder von Sprachen J.R.R. Tolkien hat in seinem großen Roman „Der Herr der Ringe“ an markanter Stelle die Fiktion einer Sprache angedeutet, die keine eigennamenhaften Komprimierungen kennt, sondern das historisch angesammelte Wissen stets in voller Länge und Wahrheit wiedergibt. Es geht um die Sprache der Baummenschen, der Wesen, die Tolkien auserkoren hat, die besten Freunde der Menschen und Hobbits zu sein, ihnen in entscheidenden Schlachten gegen das Böse zu helfen und immer die Wahrheit zu sagen. Der Baummensch Treebeard sagt einmal zu einem Hobbit, seinen wahren Namen könne er nicht preisgeben bzw. aussprechen, denn die Namen der Baummenschen seien so lang wie ihre Lebensgeschichten, weil sie eben ihre Lebensgeschichten enthielten. Es wundert nicht, daß Tolkien die Fiktion einer solchen Sprache lediglich andeuten kann; denn eine solche Sprache wie die der Baummenschen läßt sich nicht ausformulieren. So können die Hobbits, als sie aus der Ferne einer Konferenz der Baummenschen beiwohnen, nichts anderes vernehmen als ein sehr lang andauerndes harmonisches, auf- und abschwellendes Summen über den Wipfeln, dessen Wohlklang ihnen als Inbegriff von Wahrheit und Menschlichkeit gilt. ${ }^{7}$

Welche Konsequenzen kann man aus all diesen Überlegungen ziehen?

Ich möchte einige Thesen formulieren, mit denen ich an meine allgemeine Eingangsthese anknüpfen möchte, daß Sprachkritik die Freiheit fördert.

${ }^{6}$ Vgl. Heringer/Öhlschläger/Strecker/Wimmer 1977, 106-125.

7 Vgl. auch die Hinweise in Wimmer 1982 b. 


\section{These:}

Die mit syntaktisch-semantischen Mitteln produzierte Komprimierung von Aussagen in nominalen Ausdrücken kommt der kommunikativen Notwendigkeit entgegen, Wissensbestände in überschaubaren Einheiten zusammenzufassen und damit in ökonomischer Weise verfügbar und vermittelbar $\mathrm{zu}$ machen. Mit dem Verfahren sind aber auch offensichtliche Nachteile verbunden, die darin liegen,

a) daß zu vermittelnde Inhalte reduziert werden, dadurch daß sie von ihren kommunikationsgeschichtlichen Entstehungs- und Entwicklungszusammenhängen abgeschnitten werden,

b) daß Inhalte normativ so sehr verfestigt werden, daß sie den tatsächlichen Bedürfnissen des Sprachwandels und des Erkenntnisfortschritts nicht mehr gerecht werden,

c) daß die Inhaltsreduzierungen und Inhaltsnormierungen manipulativ gegen alternative Sprachverwendungen und damit zugleich gegen Erkenntnisse und Interessen der entsprechenden Sprachbenutzer eingesetzt werden.

2. These

Komplementär zur reduktiven Komprimierung von Aussagen ist das ebenfalls in der Sprachfähigkeit angelegte Prinzip der freien Gestaltung von expliziten Aussagen, in denen Eigenschaften bzw. sprachliche Ausdrücke immer wieder neu bestimmten Gegenständen der Welt oder auch sprachlichen Einheiten zu- oder abgesprochen werden und in denen auf dieses Weise die Welt ständig verändert und immer wieder neu geordnet wird. Dieses Prinzip basiert wahrscheinlich letztlich gemäß einer entsprechenden Strukturierung des menschlichen Geistes auf einer rekursiv-funktionalen Zuordnung von Wissenselementen, was nichts anderes heißt, als daß über jede beliebige Aussage eine andere explizierende Aussage ermöglicht wird, so wie es in meinem Beispiel mit der zweimal wiederholten Einbettung eines Relativsatzes exemplifiziert ist. Das Prinzip stellt sicher, daß jede Aussage und jeder Aussageteil selbst wiederum zum Gegenstand einer Aussage gemacht werden, d.h. kommentiert, interpretiert, expliziert werden kann. Es ist das Prinzip der beliebig weitgehenden Explizierungsmöglichkeit, die natürlich nur immer gemäß den jeweils praktisch-pragmatischen Bedürfnissen und Erfordernissen in Anspruch genommen und ausgenützt wird.

3. These

Die allgemeine Aufgabe der Sprachkritik besteht darin, das Prinzip der Explizierbarkeit zu erforschen und in Theorie und Praxis zu vertreten. Was die praktische Seite der Sprachkritik anbelangt, bedeutet das insbesondere,

a) kommunikations- und sprachgeschichtliche Zusammenhänge zu erforschen und zu beschreiben, in denen sprachlich verfestigte Ausdruckskomplexe stehen, und damit zur Offenheit des Sprachgebrauchs beizutragen,

b) durch Beispielanalysen exemplarisch zu zeigen, mit welchen Verfahren sprachliche Inhalte unter bestimmten Interessen normativ verfestigt und als solche in der Sprachgesellschaft durchgesetzt werden können,

c) Methoden der Explizierung komprimierter und reduzierter Aussagen zu entwickeln und lehr- und lernbar zu machen, 
d) Alternativen zu sprachlichen Normierungen und Normen zu entwickeln und vorzuschlagen, die unter jeweils bestimmten politischen, gesellschaftlichen und institutionellen Bedingungen entstanden sind und daher aktuellen Erfordernissen nicht mehr gerecht werden.

Die Sprachkritik dient nach dieser Bestimmung der Förderung von Freiheiten, die in jedermanns Sprachfähigkeit angelegt sind, und zwar von Freiheiten der Auslegung, Deutung, Veränderung und Festlegung von Bedeutungen sprachlicher Ausdrücke gemäß den individuellen Interessen und gemäß dem, was das Sprachsystem an Möglichkeiten zuläßt. Da die Gestalt der physikalischen und der sozialen Welt sowie die Struktur und Inhalte der Erkenntnis nicht unabhängig von diesen sprachlichen Potenzen sind, bedeutet die Wahrnehmung sprachkritischer Freiheiten zugleich die Wahrnehmung fundamentaler Freiheiten der Lebensgestaltung. Die Arbeit der Sprachkritik trifft sich in ihren Zielen und Aufgaben hier mit der Arbeit des Schriftstellers und Literaten, sofern diese Arbeit speziell auf die sprachliche Formung ausgerichtet ist. Ich zitiere aus dem Vortrag „Sprachkultur und Literatur“, den Adolf Muschg 1984 auf der Jahrestagung des Instituts für deutsche Sprache gehalten hat:

„... wenn es am Ende zutrifft, daß die literarische Sprache in spielender Form Handlungsmöglichkeit speichert, zuerst die Möglichkeit anders, unvorhergesehen zu handeln - wenn das wahr ist (und ich glaube, es bleibt wahr), so hätte die Sprachpflege, verstanden als alternative Kulturtechnik, heute stärkere Gründe als jemals, sich an der Literatur zu orientieren. Dann könnte Sprachpflege fast ein Synonym für Lebensrettung geworden sein, und zwar auf allen Ebenen, von der intim-privaten über die öffentlich-politische bis hin zur globalen. Denn die Literatur schärft, durch ihre Art des Zeichengebrauchs, den Möglichkeitssinn gegenüber dem bedrohlich oder tödlich, vor allem: stumpf und unempfindlich gewordenen Positiven. "8

Betrachtet man die beiden sprachlichen Prinzipien, die ich herauszustellen versucht habe, und die entsprechende Bestimmung der Aufgaben und Ziele der Sprachkritik, so kann man sich sehr wohl fragen, wieso sich die Sprachkritik im wesentlichen auf die Seite des Prinzips der Explizierbarkeit schlagen soll. Bedarf nicht auch die Normung von Inhalten einer ebensolchen Pflege? Die Antwort auf diese naheliegende Frage ist nicht ganz so einfach und hat mehrere Aspekte. Zum einen sind die beiden herausgestellten Prinzipien eng miteinander verbunden; und insofern bedeutet Arbeit an dem einen Prinzip immer auch Arbeit an dem anderen. Die Analyse überkomprimierter Ausdrücke kann nicht darauf verzichten, auch den Komprimierungsvorgang selbst zu analysieren, seine Nachteile, aber auch seine Vorteile herauszustellen.

8 Erscheint 1985 im Jahrbuch 1984 des Instituts für deutsche Sprache. Düsseldorf (= Sprache der Gegenwart Bd. 63). 


\section{Literatur}

Aspekte der Sprachkultur. Mitteilungen 10 des Instituts für deutsche Sprache. Redaktion: W. Teubert. Mannheim 1984.

Blanc, K. (Hrsg.), 1983, Tatort: Wort. München.

Braun, P., 1979, Tendenzen in der deutschen Gegenwartssprache. Stuttgart.

Greule, A., 1984, Sprachlenkung oder Sprachpflege? Zur Situation der germanistischen Sprachpflege heute. In: Language Problems and Language Planning 8/1 (1984), 50-63.

Heringer, H. J./Öhlschläger, G./Strecker, B./Wimmer, R., 1977, Einführung in die Praktische Semantik. Heidelberg.

Heringer, H. J. (Hrsg.), 1982, Holzfeuer im hölzernen Ofen. Aufsätze zur politischen Sprachkritik. Tübingen.

Hermanns, F., 1982, Brisante Wörter. Zur lexikographischen Behandlung parteisprachlicher Wörter und Wendungen in Wörterbüchern der deutschen Gegenwartssprache. In: H. E. Wiegand (Hrsg.): Studien zur neuhochdeutschen Lexikographie II. Hildesheim/New York 1982 (= GL 3-6/80), 87-108.

Ising, E. (Hrsg.), 1977, Sprachkultur - warum, wozu? Aufgaben der Sprachkultur in der DDR. Leipzig.

Mauthner, F., 31923, Beiträge zu einer Kritik der Sprache. 3 Bde. Leipzig.

Nunn, A.D., 1974, Politische Schlagwörter in Deutschland seit 1945. Ein lexikographischer und kritischer Beitrag zur Politik. Gießen.

Polenz, P. von, 91978, Geschichte der deutschen Sprache. Berlin, New York.

Polenz, P. von, 1980, Möglichkeiten satzsemantischer Textanalyse. In: Zeitschrift für germanistische Linguistik 8, 133-153.

Polenz, P. von, 1981, Über die Jargonisierung von Wissenschaftssprache und wider die Deagentivierung. In: Th. Bungarten (Hrsg.), Wissenschaftssprache. München, 85-110.

Polenz, P. von, 1983, Die Sprachkrise der Jahrhundertwende und das bürgerliche Bildungsdeutsch. In: Sprache und Literatur in Wissenschaft und Unterricht (SuL), 14. Jg., H. 52, 3-13.

Polenz, P. von, 1984, Entwicklungstendenzen des deutschen Satzbaus. In: Die deutsche Sprache der Gegenwart. Veröffentlichungen der Joachim-Jungius-Gesellschaft der Wiss. 51, Hamburg, 29-42.

Polenz, P. von, 1985, Deutsche Satzsemantik. Grundbegriffe des Zwischen-den-Zeilen-Lesens. Berlin, New York (= Sammlung Göschen 2226).

Schwenger, H., 1983, Im Jahr des großen Bruders. Orwells deutsche Wirklichkeit. München/ Zürich.

Strecker, B., 1983, Das Geschäft der Sprachkritik und die Verantwortung des Sprachwissenschaftlers. In: M. Geier, H. Wötzel (Hrsg.), Das Subjekt des Diskurses. Beiträge zur sprachlichen Bildung von Subjektivität und Intersubjektivität. Berlin (= Argument - Sonderband AS 98) $1983,7-27$.

Wimmer, R., 1982 a, Überlegungen zu den Aufgaben und Methoden einer linguistisch begründeten Sprachkritik. In: Heringer (Hrsg.), 1982, 290-313.

Wimmer, R., 1982 b, Aus Namen Mythen machen. Zu J. R. R. Tolkiens Konstruktion fiktionaler Welten. In: E. Lämmert (Hrsg.), Erzählforschung. Stuttgart 1982, 552-567.

Wimmer, R., 1983, Sprachkritik und reflektierter Sprachgebrauch. In: Sprache und Literatur in Wissenschaft und Unterricht (SuL), 14. Jg., H. 51, 3-14.

Wimmer, R., 1984, Sprachkultivierung durch Sprachkritik: Ein Plädoyer für reflektierten Sprachgebrauch. In: Aspekte der Sprachkultur. Mitteilungen 10 des Instituts für deutsche Sprache, $7-28$. 